\title{
Numerical investigation of thermocapillary migration of the drop for large Marangoni numbers
}

\author{
GAO Peng, YIN ZhaoHua \& HU WenRui ${ }^{\dagger}$ \\ National Microgravity Laboratory, Institute of Mechanics, Chinese Academy of Sciences, Beijing 100080, \\ China
}

An axisymmetric model is adopted to simulate the problem of unsteady drop thermocapillary motion for large Marangoni numbers. Front tracking methods are used in the investigation. It is found that the non-dimensional drop migration velocity will decrease with increasing Marangoni number. This agrees well with the experimental results obtained from the 4th Shen-Zhou space ship. In the meanwhile, this is also the first time for numerical simulations to verify the experimental phenomenon under large Marangoni numbers.

thermocapillary migration of drops, microgravity, front tracking methods

When an immiscible drop or a gas bubble is placed in a liquid with uniformed temperature gradient, it will move in the direction of the temperature gradient due to thermocapillary force. Such motion is termed thermocapillary migration or Marangoni migration. The thermocapillary migration of drops is an important topic in the micro-gravity fluid science with wide applications in space material science, chemical engineering, and biologic research. The pioneering work in ref. [1] used a linear model to get the analytical migration velocity of drop $\left(V_{\mathrm{YGB}}\right)$. Later works adopted the matched asymptotic method to solve the weak non-linear form of the problem (e.g., ref. [2]). The investigations considering full non-linear format mostly relied on the numerical simulation method, and only some unsteady model with a small coefficient range $(M a<100)$ and steady model work with large Ma were investigated (ref. [3] and references therein). Both experimental and numerical results showed that the non-dimensional migration velocity of the drop will decrease with increasing $M a$ when $M a<100$; but theoretical solutions were inconsistent with the space experiments beyond this range. In this paper, we studied the unsteady problem of the drop thermocapillary migration with large Ma using numerical simulations. Our simulation

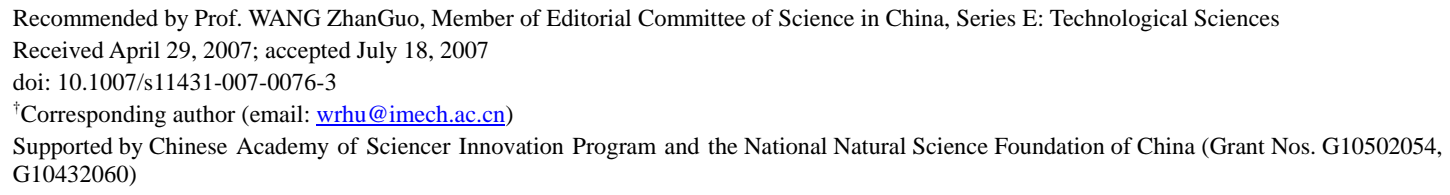


adopted the parameter of the experiments on the Chinese 4th Shen-Zhou space ship (2002), in which Fluorinert liquid FC-75 was adopted as drop liquid and 5cst silicon oil as continuous liquid with $M a$ up to $5500^{[4]}$.

In the calculation, we used an axisymmetric model to simulate the unsteady migration process of the drop from rest to the stable state. To deal with the drop interface problem, the front-tracking method was employed ${ }^{[5]}$. To validate our code, we adopted very small $R e$ and $M a$ in our simulation, and the final computed scaled migration velocities were compared with the analytical results of YGB (figure 1). The migration velocity of the drop increased from zero until it reached a steady value at last. A converging trend towards the YGB theoretic value was found when increasing resolutions in our simulations.

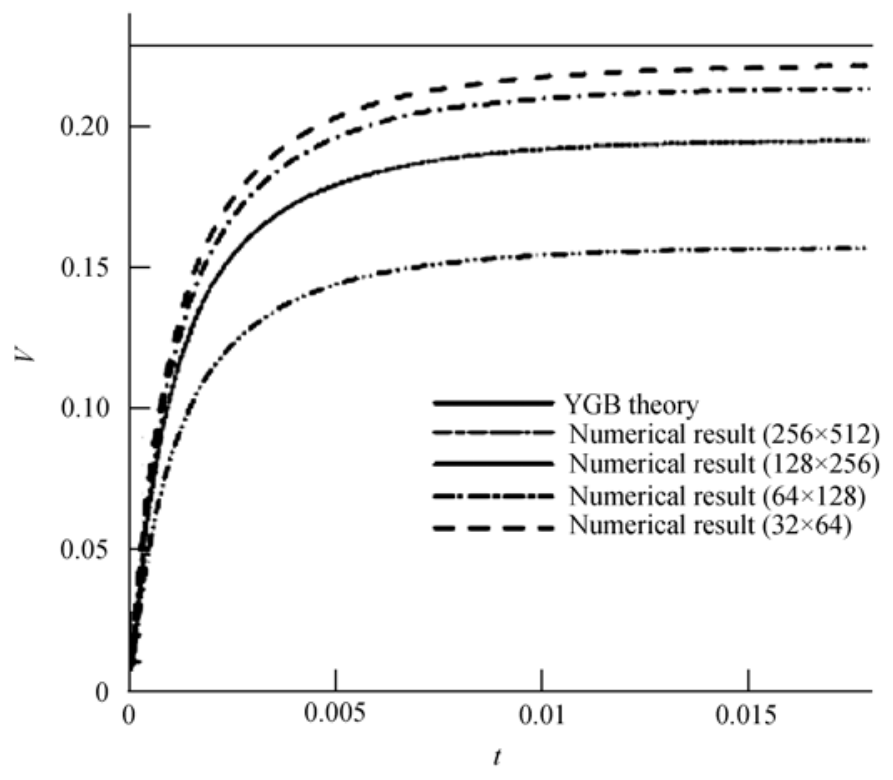

Figure 1 Comparison between theory and numerical results.

In the 4th Shen-Zhou space ship experiments, the range of Reynolds number $(R e)$ was [1.3, 51.2], and that of $M a$ was [73.8, 2813.9]. The large $M a$ means the stronger heat convection effect, thus the heat transfer would be more important in the present study, and the migration process of the drop would be very different from the small Ma cases. Figure 2 is the migration velocities of the drops versus time for five sets of non-dimensional values. When the Ma was large, after a short period of increase at the beginning of the simulation, the migration velocities start to drop until they reached a steady state. Moreover, larger Ma could lead to smaller peak values and smaller stable values of the drop migration velocities. The main reason of the overshot phenomenon of the migration velocity is the evolution of temperature field inside the drop. The cold part of fluid originally at the bottom of the drop will rise to the top by the heat convection effect, and it will reduce the temperature on the top of the drop. The change of the temperature distribution on the interface of the drop will influence the interface tension distribution, and it can finally lead the migration velocity to change. Figure 3 is the non-dimensional drop migration velocity versus $M a$. Both results show that the non-dimensional drop migration velocities would decrease with increasing $M a$. There are two reasons why there is difference between values of experiments and numerical simulations: a) the max migration velocities were sampled in the experiments, 
while the numerical results used the stable values; b) in experiments, the viscosity varied with the temperature but was treated as a constant in the numerical simulations, which also reduced the migration velocity of the drop.

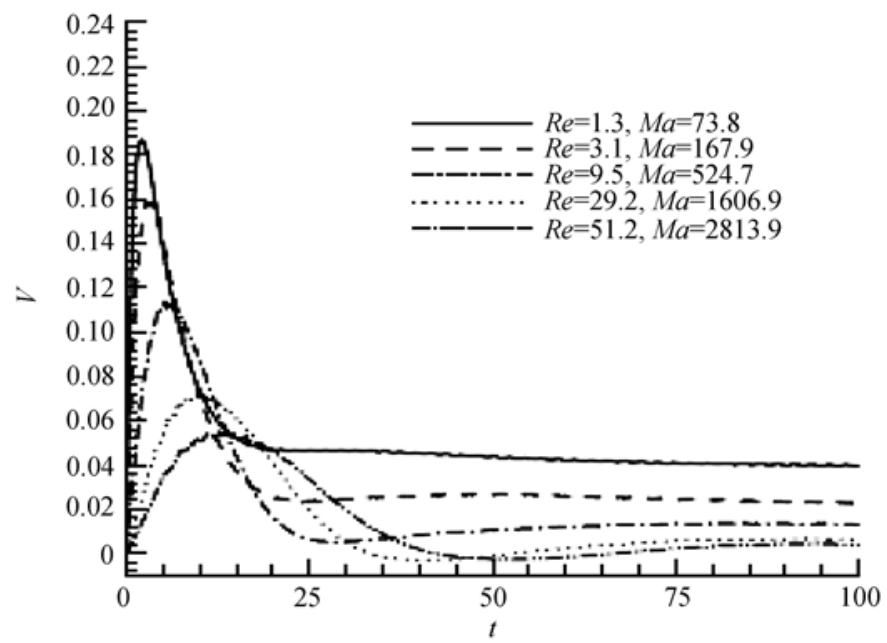

Figure 2 The migration velocities of the drops versus time.

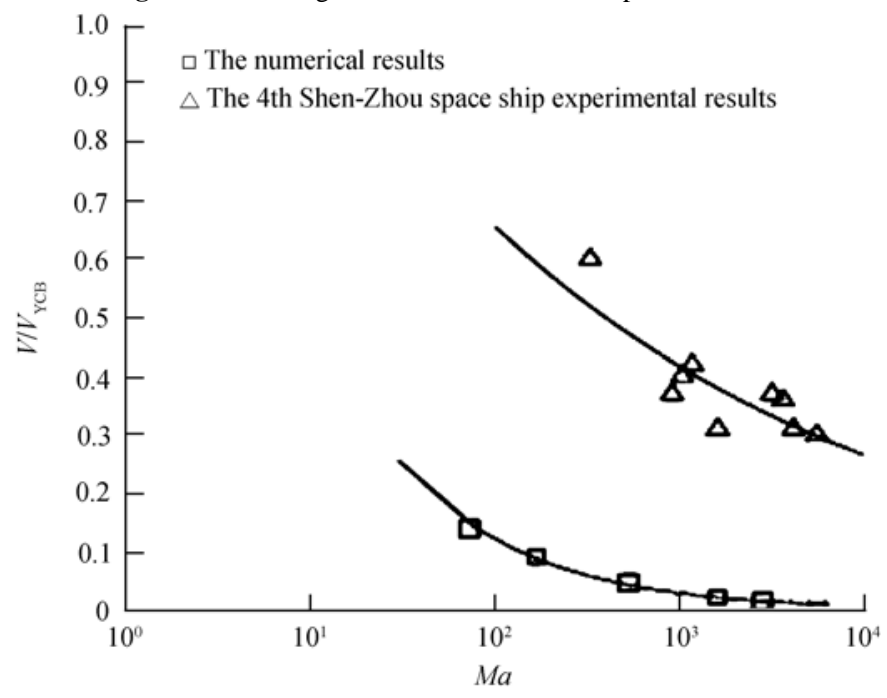

Figure 3 The non-dimensional drop migration velocity versus $M a$.

To sum up, our numerical simulations have proved that the non-dimensional drop migration velocity will decrease with increasing Ma. For the first time, our numerical work verified the experimental results for large $M a$.

1 Young N O, Goldstein J S, Block M J. The motion of bubbles in a vertical temperature gradient. J Fluid Mech, 1959, 6: $350-356$

2 Balasubramaniam R, Subramanian R S. The migration of a drop in a uniform temperature gradient at large Marangoni numbers. Phys Fluids, 2000, 12(4): 733-743[DOI]

3 Haj-Hariri H, Shi Q, Borhan A. Thermocapillary motion of deformable drops at finite Reynolds and Marangoni numbers. Phys Fluids, 1997, 9(4): 845-855[DOI]

4 Xie J C, Lin H, Zhang P, et al. Experimental investigation on thermocapillary drop migration at large Marangoni number in reduced gravity. J Colloid Interface Sci, 2005, 285: 737-743[DOI]

5 Tryggvason G, Bunner B, Esmaeeli A, et al. A front-tracking method for computations of multiphase flow. J Comput Phys, 2001, 196: 708-759[DOI] 